\title{
Enfermedades transmitidas por vectores y cambio climático
}

\author{
Vector borne disease and climate change
}

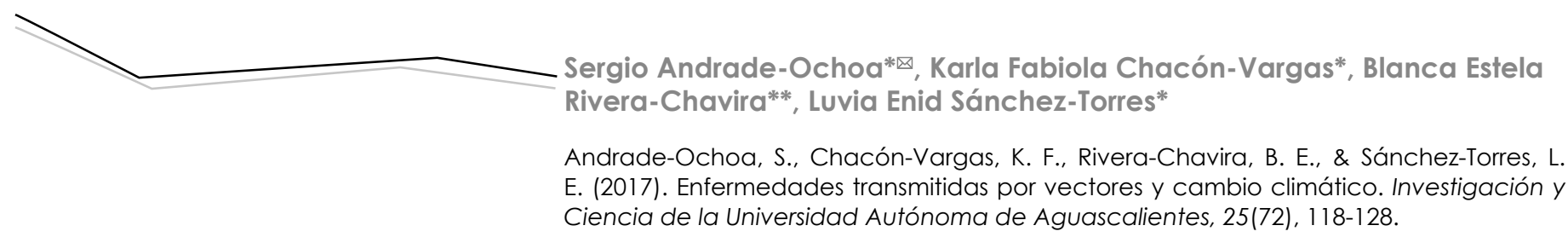

Los cambios socioeconómicos y las intervenciones sanitarias han mejorado la salud de la población mundial en las últimas décadas. Sin embargo, han empezado a presentarse modificaciones en la distribución de ciertas enfermedades debidas, principalmente, a lo que se conoce como cambio climático y que es una consecuencia más del deterioro del medio ambiente.

Una de las preocupaciones derivadas de lo anterior es la redistribución o nueva geolocalización de las enfermedades transmitidas por vectores (ETV), ya que los vectores que las transmiten están encontrando nuevos nichos ecológicos en los cuales establecerse y multiplicarse. El objetivo de la presente revisión fue conjuntar la evidencia científica en torno al cambio climático y sus efectos en las ETV.

Palabras clave: insectos; cambio climático; distribución geográfica; enfermedades transmitidas por vectores; salud pública; ecología.

Keywords: insects; climate change; geographical distribution; vector-borne disease; public health; ecology.

Recibido: 24 de octubre de 2016, aceptado: 24 de marzo de 2017

* Escuela Nacional de Ciencias Biológicas, Instituto Politécnico Nacional Unidad Profesional Lázaro Cárdenas. Prolongación de Carpio y Plan de Ayala s/n, Col. Santo Tomas, C. P. 11340, Delegación Miguel Hidalgo, México, D. F., México. Correo electrónico: s_andrade_rat@hotmail.com; karla.fabiola@live.com.mx; luviasanchez@hotmail.com

** Facultad de Ciencias Químicas, Universidad Autónoma de Chihuahua. Circuito Universitario s/n, Campus Universitario II, C. P. 31170, Chihuahua, Chihuahua, México. Correo electrónico: bchavira@uach.mx

Autor para correspondencia
Socioeconomic changes and health interventions have improved human health worldwide in recent decades. However, changes in the distribution of certain diseases occuring, due mainly to what is known as climate change, a consequence of the deterioration of our environment.

One of the concerns arising from this, is the new redistribution or geolocation of Vector-Borne Diseases (VBD) as vectors that transmit them are finding new ecological niches in which to settle and multiply. The aim of this review was to compilate the scientific evidence on climate change and its effects on VBD.

\section{INTRODUCCIÓN}

Durante el siglo XX la concentración de los gases en la atmósfera, en particular del dióxido de carbono $\left(\mathrm{CO}_{2}\right)$, ha aumentado paulatinamente como resultado de la actividad humana. En el último siglo hubo un incremento de $0.6^{\circ} \mathrm{C}$ en la temperatura promedio de la superficie terrestre (Pabón \& Nicholls, 2005), fenómeno que se mantendrá si la actividad humana persiste al ritmo actual. A este respecto, el Grupo Intergubernamental de Expertos sobre Cambios Climáticos (IPCC, por sus siglas en inglés) estimó que la temperatura media mundial se elevará varios grados celsius durante este siglo. Esta estimación genera una incertidumbre inevitable sobre el futuro desarrollo de la humanidad (Haines, Kovats, Campbell-Lendrum, \& Corvalan, 2006).

Los seres humanos, históricamente, se han beneficiado de una extensa serie de servicios ofrecidos por los ecosistemas y, por tanto, sus alteraciones producen efectos directos sobre nuestro 
estilo de vida. Se estima que el mayor efecto en la salud del cambio climático se verá reflejado en las enfermedades transmitidas por vectores (ETV) (Cerda Lorca, Valdivia, Valenzuela, \& Venegas, 2008).

Las ETV son todas aquellas enfermedades que se transmiten por medio de un hospedero intermedio al que llamamos vector, el cual usualmente es un insecto. El informe de la Organización Mundial de la Salud (OMS) de 2014, reconoce que las ETV representan más de $17 \%$ de todas las enfermedades infecciosas, y provocan cada año más de 1 millón de defunciones alrededor del mundo (Parham et al., 2015).

Durante las últimas décadas, las actividades antropogénicas, tales como la deforestación, la construcción de presas y carreteras, el cambio de uso del suelo, entre otros, han producido alteraciones en la biodiversidad de los ecosistemas, lo que modifica de manera significativa el número y movimiento de los reservorios y de los vectores e impacta directamente en la incidencia de las enfermedades en humanos (Laniak et al., 2013). Los rangos geográficos de ciertos vectores $y$, por ende, de las enfermedades que transmiten, se han ido modificando debido al cambio climático (Shuman, 2010). Además, los eventos climáticos extremos crean condiciones que conducen a brotes de enfermedades infecciosas, y a esto se deben sumar, las malas condiciones sanitarias en la gestión de residuos, la falta de sistemas de almacenamiento y de suministro de agua, que en su conjunto, favorecen la reproducción y aumento de la población, principalmente de mosquitos (Laniak et al., 2013; Quintero et al., 2014).

Aunado a la problemática del cambio climático se debe considerar que el uso intensivo de insecticidas sintéticos en el control de mosquitos y otros insectos ha tenido efectos indeseables sobre organismos no específicos que conviven con los vectores, así como influencia en la vida silvestre, daños a la salud humana (Miranda et al., 2003) y el desarrollo de mosquitos vectores resistentes (Ocampo, Salazar-Terreros, Mina, McAllister, \& Brogdon, 2011).

Actualmente se han utilizado diversos modelos matemáticos para facilitar la comprensión de las complejas interacciones entre las poblaciones de vectores y sus hospederos (Dunn, Davis, Stacey, \& DiukWasser, 2013; Ludwig, Ginsberg, Hickling, \& Ogden, 2015; Nonaka, Ebel, \& Wearing, 2010). La simulación de escenarios permite el estudio de hipótesis generadas en la investigación de campo y da una idea de las observaciones empíricas aparentemente contraintuitivas o contrastantes. Estos modelos permiten, a través de la identificación y evaluación de los parámetros clave en la transmisión, sintetizar el conocimiento actual para indicar las prioridades en las investigaciones y perspectivas futuras.

Con la finalidad de identificar el conjunto de factores ambientales que explican la distribución actual de los vectores se han utilizado modelos fenomenológicos, los cuales suponen que las condiciones climáticas representan todo el hábitat adecuado en donde los vectores pueden desarrollarse. Posteriormente, este conjunto de condiciones permite estimar las futuras distribuciones del vector o de la enfermedad (Ostfeld \& Brunner, 2015). Los modelos mecanicistas, por su parte, tienen la ventaja de ser capaces de proyectar mecanismos biológicos, a diferencia de las simples asociaciones. La enorme cantidad de parámetros climáticos y demográficos relacionados con las variables de respuesta y de comportamiento plantean enormes desafíos para la construcción y la comprensión de las ETV (Ludwig et al., 2015). Los modelos mecanicistas consisten en desmenuzar los fenómenos complejos en partes, para comprender, desde las propiedades de las mismas, el funcionamiento del todo. La mayoría de los modelos mecanicistas, por tanto, permiten conocer los principales factores que se creen dominantes (Estrada-Peña, Ostfeld, Peterson, Poulin, \& de la Fuente, 2014).

Otros modelos, de reciente uso, son los de la matriz de la próxima generación, los cuales han resultado ser una sencilla forma de evaluar la sensibilidad, o el riesgo, de una población. El enfoque de estos modelos es obtener simulaciones a partir del número de casos de individuos infectados en una población totalmente susceptible (RO), posteriormente se puede determinar la tendencia lineal a partir de parámetros extraídos de la literatura, ya sean antecedentes, actuales o predicciones (Roberts \& Heesterbeek, 2013). Los modelos de matriz de la próxima generación han sido epidemiológicamente útiles para determinar la tasa y persistencia de infección (Nonaka et al., 2010), así como el éxito que pueden tener algunos vectores para invadir y/o propagarse (Dunn et al., 2013).

A continuación se describen los resultados de diversos modelos matemáticos sobre la relación del cambio climático y las principales ETV a nivel mundial. 


\section{Dengue}

El modelado formal de los efectos del cambio climático en las ETV se ha centrado en el paludismo y el dengue, siendo el de esta enfermedad viral más simple. Mientras que el paludismo conlleva principalmente dos especies de parásito (Plasmodium falciparum y Plasmodium vivax) y se basa en varias docenas de especies de mosquitos, la transmisión de la fiebre por dengue depende principalmente de un mosquito vector, Aedes aegypti, y en menor medida en Aedes albopictus (Murray, Quam, \& Wilder-Smith, 2013).

El mosquito vector requiere agua para reproducirse y de una temperatura ambiente cálida para el desarrollo de las larvas y la replicación del virus (Banu, Hu, Hurst, \& Tong, 2011). La incidencia de dengue ha aumentado significativamente en los últimos 35 años y varios factores, entre ellos la falta de servicios básicos como agua potable, drenaje y luz, la globalización y el cambio climático, han sido los principales contribuyentes (Hopp \& Foley, 2001).

Estudios recientes han demostrado una asociación entre la variabilidad del clima y el dengue (Chen, \& Hsieh, 2012; Hu, Clements, Williams, Tong, \& Mengersen, 2012). Han encontrado que la temperatura, las precipitaciones y la humedad están directamente asociadas con su transmisión (Karim, Munshi, Anwar, \& Alam, 2012). La temperatura influye en el ciclo de vida de los mosquitos Aedes, incluyendo tasa de crecimiento, la supervivencia de las larvas, huevos y la longitud del ciclo reproductivo. Se ha observado que una tasa de supervivencia de $88-93 \%$ de mosquitos se encuentra entre $20-30^{\circ} \mathrm{C}$ (Tun-Lin, Burkot, \& Kay, 2000). Este factor también afecta a la replicación del virus, la maduración y el período de infectividad (Yang, Macoris, Galvani, Andrighetti, \& Wanderley, 2009).

Algunos trabajos en países asiáticos han demostrado que el dengue se incrementará más de 40 veces en el año 2100 con relación al año 2010, cuando la temperatura ambiente aumente $3.3^{\circ} \mathrm{C}$, de acuerdo con la proyección del clima regional del IPCC (Banu et al., 2011). El continente asiático es el más afectado en cuanto al número de personas infectadas y la distribución del vector, ya que se caracteriza por grandes extensiones de zonas densamente pobladas que presentan las condiciones idóneas para la transmisión de la enfermedad. Tan sólo en la India se presentaría 34\% de infecciones (24-44 millones más infecciones de lo reportado en 2010) a nivel mundial (Chakravarti, Arora, \& Luxemburger, 2012; Kakkar, 2012).
Predicciones realizadas por Campbell et al. (2015) basadas en las proyecciones para 2050 han concluido que existe una potencial expansión de Ae. aegypti hacia el este de América del Norte, el sur de América del Sur y el este de Asia. En México se predice una potencial expansión de Ae. aegypti en la zona del Valle de México, Sonora, Chihuahua y Durango, mientras que para Ae. albopictus se predice su potencial expansión en Veracruz y Tamaulipas (figura 1), además de un aumento drástico en la presencia de estos vectores en el sureste de los Estados Unidos (Campbell et al., 2015). Sin embargo, un análisis reciente indica que Ae. albopictus no parece conducir a grandes brotes de dengue (Lambrechts, Scott, \& Gubler, 2010), tanto el patrón de ocurrencias como en mapas de distribución actual y potencial para las dos especies sugieren que Ae. aegypti emerge como el principal impulsor de la transmisión del dengue, por lo que los papeles relativos y la importancia de las dos especies en la transmisión del virus parecen ser desiguales (figura 1).

Cambios en la distribución de Ae. aegypti y de Ae. albopictus (Radke et al., 2012) podrían ampliar la distribución de otras enfermedades de transmisión por Aedes, como el Chikungunya o el Zika, que se transmiten fácilmente por Ae. albopictus (Tsetsarkin, Chen, Sherman, \& Weaver, 2011), padecimientos que han demostrado una reciente expansión geográfica (Fischer et al., 2013; Van Bortel et al., 2014).

\section{Leishmaniasis}

La leishmaniasis es un complejo de enfermedades de mamíferos causadas por protozoos parásitos del género Leishmania (WHO, 2009). La transmisión es mediante la mordedura de una mosca, de los géneros Phlebotomus (Viejo Mundo) y Lutzomyia (Nuevo Mundo) (Ready, 2008). A nivel mundial, al menos 20 especies de Leishmania causan leishmaniasis cutánea (LC) y leishmaniasis visceral (LV) humana (Singh, Kumar, \& Singh, 2012).

De igual manera que para otros vectores, la mayoría de los modelos de predicción para los flebótomos se basan en los cambios de temperatura esperados para los siguientes 100 años. Algunos modelos han reportado que la población de estos vectores podría aumentar en el norte de Europa (de La Rocque, Rioux, \& Slingenbergh, 2008), pues se ha sugerido que las temperaturas más altas pueden inducir el vuelo de los insectos adultos (Hufnagel \& Kocsis, 2011). Se ha encontrado que la elevación de 


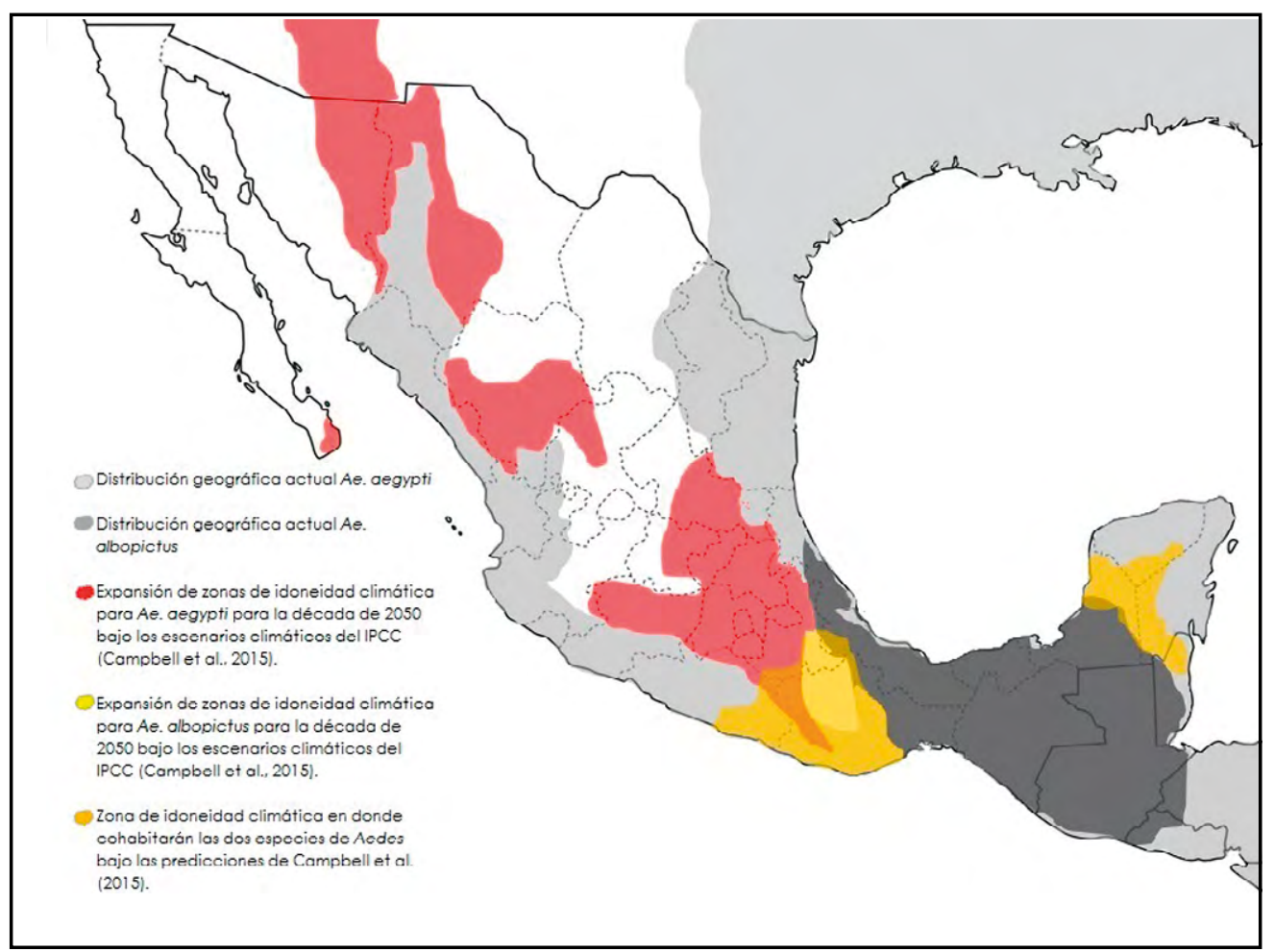

Figura 1. Modelado de distribución geográfica de Ae. aegypti y Ae. albopictus en la República Mexicana.

Elaboración propia.

la temperatura incrementa significativamente la proporción general de flebótomos infectados. El calor, la humedad y suficiente materia orgánica son los principales factores para su desarrollo (Lindgren \& Naucke, 2006), mientras que los niveles de $\mathrm{CO}_{2}$ crecientes son generalmente factores desfavorables para el desarrollo de las larvas (Mencke, 2011). La leishmaniasis es considerada una enfermedad emergente en Europa y un signo de este aumento es la presencia de focos de infección en perros. Modelos de idoneidad climática sugieren que el vector y la leishmaniasis llegarán a finales del siglo XXI a Europa (Fischer, Thomas, \& Beierkuhnlein, 2011), inclusive la presencia de vectores no endémicos ya se ha reportado (Farkas et al., 2011 ; Tánczos et al., 2012; Trájer et al., 2013).

Por otro lado, Leishmania mexicana es responsable de la mayoría de los casos cutáneos humanos de leishmaniasis en América del Norte. A lo largo de la frontera México-Estados Unidos, la forma cutánea de la enfermedad se encuentra en hábitats de matorral semiáridos desde Sonora hasta Tamaulipas, y son las especies Lutzomyia diabólica y Lutzomyia anthophora los vectores probables (Wright, Davis, Aftergut, Parrish, \& Cockerell, 2008). Por otro lado, la distribución conocida de Lutzomyia olmeca en México, hasta el presente, comprende a los estados de Campeche, Yucatán, Quintana Roo, además de Tabasco, Chiapas, Oaxaca y Veracruz (González et al., 201 1; Rebollar-Téllez, Tun-Ku, Manrique-Saide, \& Andrade Narvaez, 2013). González et al. (2010) han previsto una potencial expansión de Lu. diabólica y de Lu. anthophora para los años 2020, 2050 y 2080; destaca que si las predicciones del aumento del clima se cumplen para 2080, Lu. diabólica podría distribuirse hasta el sureste de Canadá, invadiendo todo el centro y este de Estados Unidos y prácticamente todo México (figura 2). Un estudio realizado por Pigott et al. (2014) estima que de cumplirse las predicciones en el cambio climático, 1,710 millones de individuos estarán en riesgo de contraer LC y 1,690 millones LV. Sin embargo, en algunos países, como México, en donde la enfermedad no es de notificación obligatoria, existe un importante subregistro, por lo que las predicciones basadas en evidencia tenderán a ser datos subestimados.

\section{Tripanosomiasis}

Los insectos vectores de Trypanosoma cruzi, el agente etiológico de la tripanosomiasis americana, 


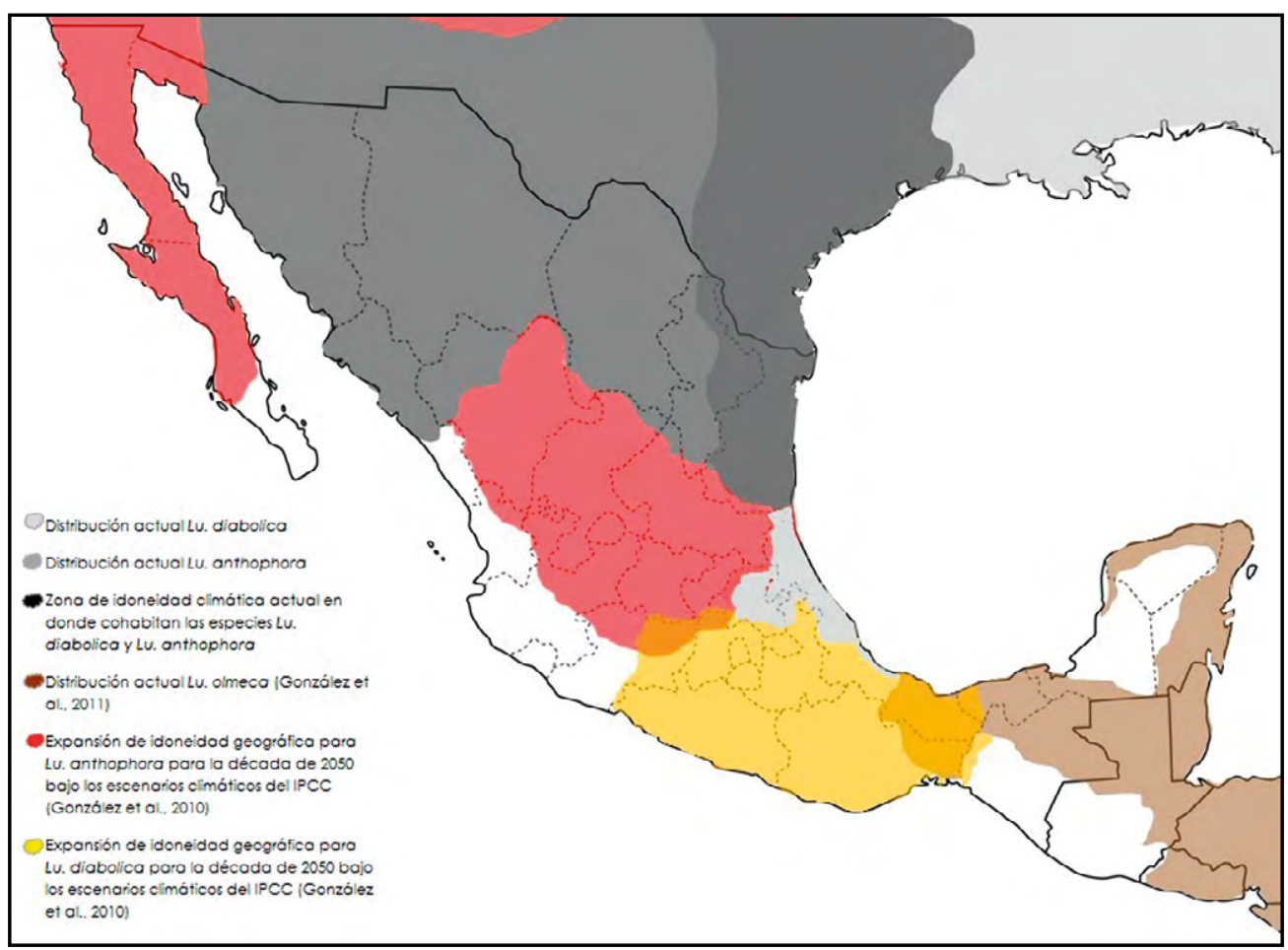

Figura 2. Modelado de distribución geográfica de Lu. diabólica, Lu. anthophora y Lu. olmeca. Elaboración propia.

también conocida como la enfermedad de Chagas, pertenecen al género Triatoma que contiene más de 140 especies, agrupadas en 18 géneros (Schofield \& Galvão, 2009). Con pocas excepciones, los triatominos tienen estricto régimen de alimentación hematófaga basado principalmente en sangre de aves y mamíferos, incluidos roedores, así como de seres humanos (Coura, 2013). Los triatominos habitan una gran variedad de ambientes y están bien adaptados a una gran variedad de climas.

Aunque la mayoría de las especies de triatominos se distribuyen en zonas intertropicales, también llegan a las regiones templadas con inviernos fríos como la Patagonia, Indiana y Maryland. Desde una perspectiva epidemiológica, Rhodnius prolixus (en Centroamérica y el norte de América del Sur) y Triatoma infestans (en la región del cono Sur de América del Sur) son las especies de vectores más importantes (Guhl, 2009); el primero está adaptado a las regiones tropicales, mientras que el segundo a sitios templados. El complejo Triatoma brasilensis es un grupo monofilético también de importancia debido a que esta especie presenta una de las tasas de infección de T. cruzi más altas, así como elevados índices de infestación intradomiciliarios (Ceballos et al., 2011).
Los efectos de las variables ambientales, como temperatura y precipitación, sobre los procesos fisiológicos y de comportamiento, han sido ampliamente demostrados para $R$. prolixus y $T$. infestans (Ferreira, Lazzari, Lorenzo, \& Pereira, 2007). En particular, la temperatura se ha relacionado con los procesos térmicos de preferencias, así como el encuentro con un hospedero (Fresquet \& Lazzari, 2011), la alimentación, la producción de huevos, la tasa de eclosión, el tiempo de desarrollo, el cese de los procesos de muda y la tasa metabólica (Abad-Franch, Diotaiuti, GurgelGonçalves, \& Gürtler, 2013). Por tanto, el cambio climático tiene efectos sobre los procesos vitales $y$, en consecuencia, sobre el potencial de la distribución geográfica de ambas especies.

Varios autores han señalado que la situación actual podría modificarse en virtud de futuros escenarios epidemiológicos que implican cambios en la distribución e infraestructura de las urbes, el aumento de vectores relictos y el cambio climático (Abad-Franch et al., 2013). Medone, Ceccarelli, Parham, Figuera, \& Rabinovich (2015) calcularon que existirá un impacto diferencial del cambio climático en las dos especies de vectores para el 2050: $R$. prolixus muestra una futura expansión a nuevas áreas, mientras que $T$. infestans muestra una disminución en su área de distribución geográfica. Estos resultados 


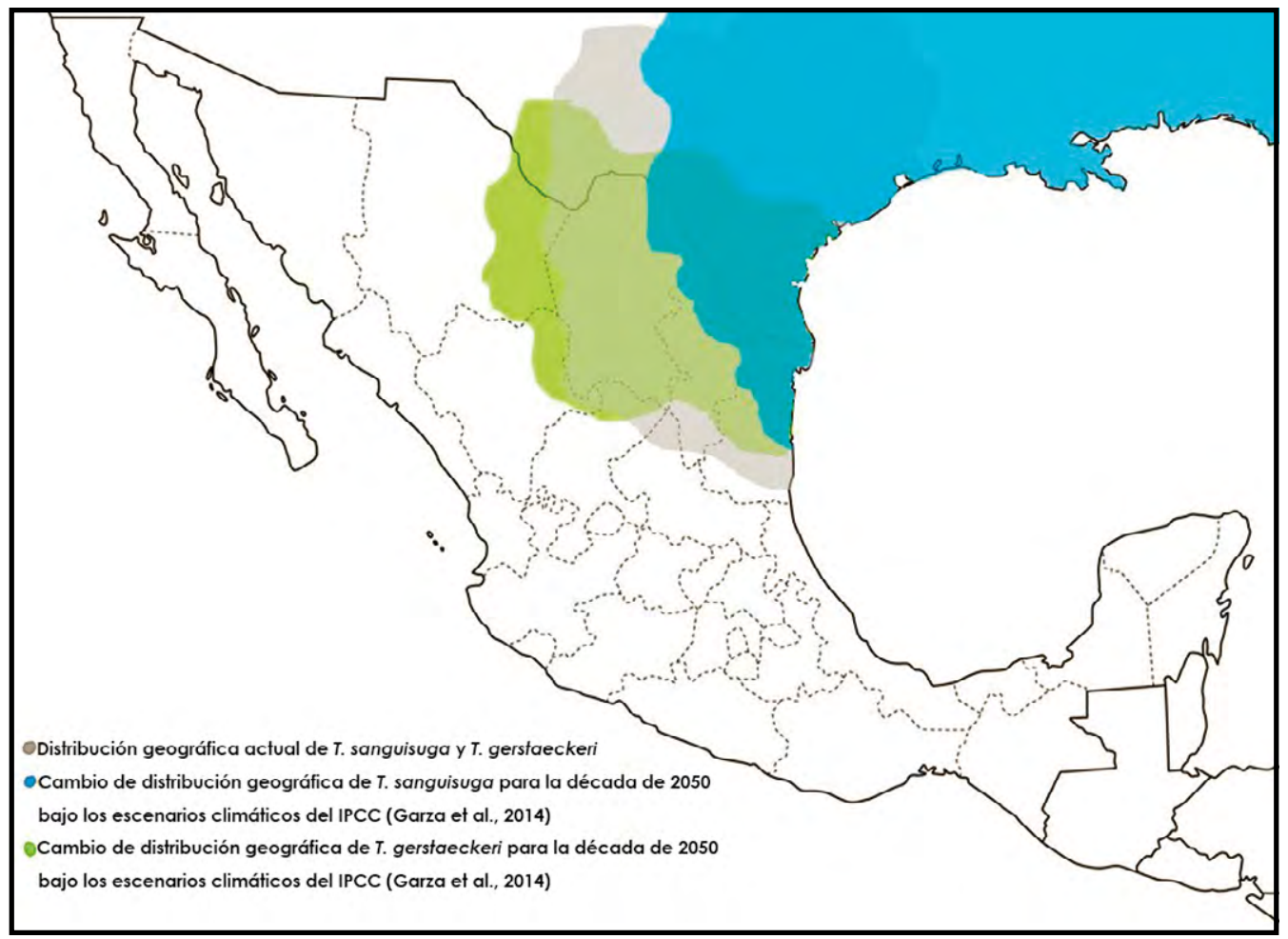

Figura 3. Modelado de distribución geográfica de T. sanguisuga y T. gerstaeckeri.

Elaboración propia.

sugieren que la enfermedad tendría un cambio de localización para 2050 y no una expansión (Rolandi \& Schilman, 2012). Garza et al. (2014) concuerdan en que para 2050 la distribución en América del Norte de Triatoma gerstaeckeri y de T. sanguisuga se ampliará desde los estados de Veracruz, Coahuila y Tamaulipas hasta Michigan y Nueva York (figura 3).

\section{Borreliosis de Lyme y rickettsiosis}

El incremento de casos de borreliosis (comúnmente conocida como enfermedad de Lyme) en la última década ha promovido su clasificación como un padecimiento infeccioso emergente (Margos, Vollmer, Ogden, \& Fish, 2011). En 2012 se reportaron 30,831 casos en Estados Unidos (Feria-Arroyo et al., 2014) y se estima que 300,000 personas se infectan anualmente (Kuehn, 2013). Varias especies de garrapatas del género Ixodes han sido reconocidas como vectores de la espiroqueta Borrelia burgdorferi, el agente causal de la enfermedad (Stanek, Wormser, Gray, \& Strle, 2012). Ixodes scapularis e I. pacificus son los vectores más comunes en México y Estados Unidos, mientras que $I$. persulcatus e I. ricinus son las especies más frecuente documentadas en Europa y Asia (Mannelli, Bertolotti, Gern, \& Gray, 2012).
Modelos de simulación mecanicista del ciclo de vida I. scapularis han identificado efectos de la temperatura sobre la supervivencia de la garrapata con el fin de ayudar en la evaluación de riesgo y futuro de la enfermedad de Lyme en Canadá (Ogden et al., 2006). Estudios de campo prospectivos y análisis retrospectivos sobre la garrapata y el patógeno han validado los hallazgos del modelo sobre los escenarios en el sureste de Canadá e identificaron a la temperatura como un factor determinante estadísticamente significativo (Bouchard et al., 2013). Trabajos más recientes han generado modelos de reacomodo geográfico de l. scapularis sobre climas proyectados para 2040 y 2070 en Norteamérica. Los resultados demuestran una expansión en nuevas regiones sobre del noroeste de Estados Unidos y sureste de Canadá (Ogden et al., 2014). Estos resultados concuerdan con las estimaciones realizadas por Feria-Arroyo et al. (2014) para 2050, los cuales demuestran un aumento en la distribución de I. scapularis en todo el norte de México y alta ocurrencia en todo el Golfo de México, en donde Tamaulipas y Texas son los estados de mayor probabilidad de incidencia. La precipitación y temperatura resultan las variables de mayor relevancia correlacionadas con la presencia del vector. 
Un estudio realizado en Irlanda sugiere que los veranos calientes pueden causar que las garrapatas sean más abundantes en otoño y principios de invierno (Gray, 2008). En la década pasada se predijo que la redistribución de I. ricinus podría cambiar a un patrón europeo más meridional (Estrada-Peña, Martínez, Sánchez Acedo, Quilez, \& Del Cacho, 2004). Actualmente existe considerable evidencia de que la incidencia de la enfermedad de Lyme ha aumentado en toda Europa (Fülöp \& Poggensee, 2008; Heyman et al., 2010). Un estudio sugiere que el cambio climático altera las proporciones relativas de los genotipos de $B$. burgdorferi e influye en el tiempo de maduración de larvas y ninfas de I. scapularis (Gatewood et al., 2009); de hecho, se ha demostrado que las garrapatas y ninfas de I. ricinus infectadas tienen mayor supervivencia a condiciones climáticas difíciles para ellos (Herrmann \& Gern, 2010). Estos autores informaron que la infección por B. burgdorferi o B. afzelii confiere ventajas de supervivencia en I. ricinus en condiciones termohigrométricas estresantes. Estos resultados se relacionan con el aumento en la densidad de garrapatas infectadas con Borrelia en Suiza durante el periodo 2003-2005, en donde las temperaturas promedio alcanzaron un máximo reportado (Morán Cadenas et al., 2007); de igual manera se relaciona con el aumento en la incidencia de casos de borreliosis confirmados en el 2011 en el Reino Unido (Dubrey, Bathia, Woodham, \& Rakowicz, 2014).

Otro grupo importante de agentes patógenos transmitidos por garrapatas son las bacterias del género Rickettsia, causantes de la enfermedad emergente (y re-emergente) rickettsiosis. En Europa la fiebre manchada de las Montañas Rocosas es la más grave de las rickettsiosis y es producida por Rickettsia ricketsii, la fiebre manchada del Mediterráneo es producida por $R$. conorii y es la enfermedad más común transmitida por garrapatas al norte de África; la fiebre manchada de Israel, por su parte, es considerada una enfermedad emergente (Oteo \& Portillo, 2012).

Una serie de vectores de garrapatas están involucrados en la transmisión y se sabe muy poco acer- ca de las interacciones entre estos patógenos bacterianos y sus invertebrados vectores. Rhipicephalus sanguineus es una garrapata asociada en la transmisión de $R$. conorii en el norte de África (Kernif, Socolovschi, Bitam, Raoult, \& Parola, 2012), Hyalomma asiaticum de R. sibirica en Mongolia, en Argentina se ha reportado a Amblyomma tigrinum como vector (Romer et al., 2014) y actualmente incluso las sanguijuelas son objeto de estudios como posibles vectores (Slesak, Inthalath, Dittrich, Paris, \& Newton, 2015). En México, la enfermedad se considera reemergente y se identifica a las garrapatas Rhipicephalus sanguineus y Amblyomma cajennense como los vectores transmisores de la enfermedad (Peniche-Lara, PerezOsorio, Dzul-Rosado, \& Zavala-Castro, 2015).

Debido al gran número de posibles vectores y a que la enfermedad está totalmente subdiagnosticada, actualmente se carece de modelos predictivos; sin embargo, es bien sabido que el ciclo de vida silvestre y semiurbano se mantiene en fincas de clima medio, principalmente en regiones donde existe la garrapata y, por ende, se puede inferir que los modelos centrados en la redistribución de garrapatas también pueden correlacionar la redistribución e incidencia de la enfermedad (Süss, Klaus, Gerstengarbe, \& Werner, 2008; Walker, 2016).

CONCLUSIONES

Varios grupos de investigación alrededor del mundo han generado modelos predictivos para evidenciar la redistribución e incidencia de las ETV, así como las poblaciones en riesgo y demostrar la relación directa entre el cambio climático, la falta de servicios básicos en las áreas urbanas y las ETV. En general, las predicciones sobre la redistribución de los vectores son desfavorables y representan un problema de salud pública que sobrepasa las fronteras políticas. Actualmente, México cuenta con pocos estudios de predicción en esta materia, lo que hace imperativo el desarrollo de modelos predictivos para nuestro país, que permitan, entre muchas cosas, el desarrollo de estrategias de prevención y control en las actuales zonas endémicas y en las futuras zonas en peligro. 
- Abad-Franch, F., Diotaiuti, L., Gurgel-Gonçalves, R., \& Gürtler, R. E. (2013). Certifying the interruption of Chagas disease transmission by native vectors: cui bono? The Memórias do Instituto Oswaldo Cruz, 108(2), 251-254.

- Banu, S., Hu, W., Hurst, C., \& Tong, S. (2011). Dengue transmission in the Asia-Pacific region: impact of climate change and socio-environmental factors. Tropical Medicine \& International Health, 16(5), 598-607.

- Bouchard, C. et al. (2013). Harvested white-tailed deer as sentinel hosts for early establishing /xodes scapularis populations and risk from vector-borne zoonoses in southeastern Canada. Journal of Medical Entomology, 50(2), 384-393.

- Campbell, L. P., Luther, C., Moo-Llanes, D., Ramsey, J. M., DanisLozano, R., \& Townsend Peterson, A. (2015). Climate change influences on global distributions of dengue and chikungunya virus vectors. Philosophical Transactions of the Royal Society B: Biological, 370(1665), 20140135. doi: 10.1098/rstb.2014.0135

- Ceballos, L. A., Piccinali, R. V., Marcet, P. L., Vazquez-Prokopec, G. M., Cardinal, M. V., Schachter-Broide, J.,...Gurtler, R. E. (2011). Hidden sylvatic foci of the main vector of Chagas disease Triatoma infestans: Threats to the vector elimination campaign? PLoS Neglected Tropical Diseases, 5(10), el365. doi: 10.1371/journal.pntd.0001365

- Cerda Lorca, J., Valdivia, G., Valenzuela, M. T., \& Venegas L., J. (2008). Cambio climático y enfermedades infecciosas: un nuevo escenario epidemiológico. Revista Chilena de Infectología, 25(6), 447-452.

- Chakravarti, A., Arora, R., \& Luxemburger, C. (2012). Fifty years of dengue in India. Transactions of the Royal Society of Tropical Medicine and Hygiene, 106(5), 273-282.

- Chen, S. C., \& Hsieh, M. H. (2012). Modeling the transmission dynamics of dengue fever: Implications of temperature effects. Science of the Total Environment, 431, 385-391.

- Coura, J. R. (2013). Chagas disease: control, elimination and eradication. Is it possible? Memórias do Instituto Oswaldo Cruz, $108(8), 962-967$.

- De La Rocque, S., Rioux, J. A., \& Slingenbergh, J. (2008). Climate change: effects on animal disease systems and implications for surveillance and control. Revue Scientifique et Technique (International Office of Epizootics), 27(2), 339-354.

- Dubrey, S. W., Bathia, A., Woodham, S., \& Rakowicz, W. (2014). Lyme disease in the United Kingdom. Postgraduate Medical Journal, 90(1059), 33-42.

- Dunn, J. M., Davis, S., Stacey, A., \& Diuk-Wasser, M. A. (2013). A simple model for the establishment of tick-borne pathogens of
Ixodes scapularis: A global sensitivity analysis of RO. Journal of Theoretical Biology, 335, 213-221.

- Estrada-Peña, A., Martínez, J. M., Sánchez Acedo, C., Quilez, J., \& Del Cacho, E. (2004). Phenology of the tick, Ixodes ricinus, in its southern distribution range (central Spain). Medical and Veterinary Entomology, 18(4), 387-397.

- Estrada-Peña, A., Ostfeld, R. S., Peterson, A. T., Poulin, R., \& de la Fuente, J. (2014). Effects of environmental change on zoonotic disease risk: an ecological primer. Trends in Parasitology, 30 (4), 205-214.

- Farkas, R., Tánczos, B., Bongiorno, G., Maroli, M., Dereure, J., \& Ready, P. D. (2011). First surveys to investigate the presence of canine leishmaniasis and its phlebotomine vectors in Hungary. Vector Borne and Zoonotic Diseases, 11 (7), 823-834.

- Feria-Arroyo, T.P., Castro-Arellano, I., Gordillo-Pérez, G., Cavazos, A. L., Vargas-Sandoval, M., Grover, A.,...Esteve-Gassent, M. D. (2014). Implications of climate change on the distribution of the tick vector Ixodes scapularis and risk for Lyme disease in the Texas-Mexico transboundary region. Parasites \& Vectors, 7(1), 199. Recuperado de https://doi.org/10.1186/1756-3305-7-199

- $\quad$ Ferreira, R. A., Lazzari, C. R., Lorenzo, M. G., \& Pereira, M. H. (2007). Do haematophagous bugs assess skin surface temperature to detect blood vessels? PLoS One, 2(9), e932. doi: 10.1371/journal. pone.0000932

- Fischer, D., Thomas, S. M., \& Beierkuhnlein, C. (2011). Modelling climatic suitability and dispersal for disease vectors: the example of a phlebotomine sandfly in Europe. Procedia Environmental Sciences, 7, 164-169.

- Fischer, D., Thomas, S. M., Suk, J. E., Sudre, B., Hess, A., Tjaden, N. B.,...Semenza, J. C. (2013). Climate change effects on Chikungunya transmission in Europe: geospatial analysis of vector's climatic suitability and virus' temperature requirements. International Journal of Health Geographics, 12(1), 51. Recuperado de https://doi.org/10.1186/1476-072X-12-51

- Fresquet, N., \& Lazzari, C. R. (2011). Response to heat in Rhodnius prolixus: the role of the thermal background. Journal of Insect Physiology, 57(10), 1446-1449.

- Fülöp, B., \& Poggensee, G. (2008). Epidemiological situation of Lyme borreliosis in Germany: Surveillance data from six Eastern German States, 2002 to 2006. Parasitology Research, 103(Supl. 1), S117-S120.

- Garza, M., Feria Arroyo, T. P., Casillas, E. A., Sánchez-Cordero, V., Rivaldi, C-L., \& Sarkar, S. (2014). Projected future distributions of vectors of Trypanosoma cruzi in North America under climate change scenarios. PLoS Neglected Tropical Diseases, 8(5), e2818. Recuperado de https://doi.org/10.1371/journal.pntd.0002818 
- Gatewood, A. G., Liebman, K. A., Vourc'h, G., Bunikis, J., Hamer, S. A., Cortinas, R.,...Diuk-Wasser, M. A. (2009). Climate and tick seasonality are predictors of Borrelia burgdorferi genotype distribution. Applied and Environmental Microbiology, 75(8), 2476-2483.

- González, C., Rebollar-Téllez, E. A., Ibáñez-Bernal, S., BeckerFauser, I., Martínez-Meyer, E., Peterson, A. T., \& Sánchez-Cordero, V. (2011). Current knowledge of Leishmania vectors in Mexico: How geographic distributions of species relate to transmission areas. American Journal of Tropical Medicine and Hygiene, 85(5), 839-846. doi: 10.4269/ajtmh.2011.10-0452

- González, C., Wang, O., Strutz, S. E., González-Salazar, E., Sánchez-Cordero, E., \& Sarkar, S. (2010). Climate change and risk of leishmaniasis in North America: Predictions from ecological niche models of vector and reservoir species. PLoS Neglected Tropical Diseases, 4(1), e585. Recuperado de https://doi.org/10.1371/journal.pntd.0000585

- Gray, J. S. (2008). Ixodes ricinus seasonal activity: Implications of global warming indicated by revisiting tick and weather data. International Journal of Medical Microbiology, 298, 19-24.

- Guhl, F. (2009). Enfermedad de Chagas: Realidad y perspectivas. Revista Biomédica, 20, 228-234.

- Haines, A., Kovats, R. S., Campbell-Lendrum, D., \& Corvalan, C. (2006). Climate change and human health: Impacts, vulnerability and public health. Public Health, 120(7), 585-596.

- Herrmann, C., \& Gern, L. (2010). Survival of Ixodes ricinus (Acari: Ixodidae) under challenging conditions of temperature and humidity is influenced by Borrelia burgdorferi sensu lato infection. Journal of Medical Entomology, 47(6), 1196-1204.

- Heyman, P., Cochez, C., Hofhuis, A., van der Giessen, J., Sprong, H., Porter, S. R.,...Papa, A. (2010). A clear and present danger: tick-borne diseases in Europe. Expert Review of Antiinfective Therapy, 8(1), 33-50. doi: 10.1586/eri.09.118

- Hopp, M. J., \& Foley, J. A. (2001). Global-scale relationships between climate and the dengue fever vector, Aedes aegypti. Climatic Change, 48(2-3), 441-463.

- Hu, W., Clements, A., Williams, G., Tong, S., \& Mengersen, K. (2012). Spatial patterns and socioecological drivers of dengue fever transmission in Queensland, Australia. Environmental Health Perspectives, 120(2), 260.

- Hufnagel, L., \& Kocsis, M. (2011). Impacts of climate change on Lepidoptera species and communities. Applied Ecology and Environmental Research, 9(1), 43-72.

- Kakkar, M. (2012). Dengue fever is massively under-reported in India, hampering our response. British Medical Journal, 345, e8574. doi: 10.1136/bmj.e8574
- Karim, M., Munshi, S. U., Anwar, N., \& Alam, M. S. (2012). Climatic factors influencing dengue cases in Dhaka city: a model for dengue prediction. Indian Journal of Medical Research, 136(1), 32-39.

- Kernif, T., Socolovschi, C., Bitam, I., Raoult, D., \& Parola, P. (2012). Vector-borne rickettsioses in North Africa. Infectious Disease Clinics of North America, 26(2), 455-478.

- Kuehn, B. M. (2013). CDC estimates 300000 US cases of Lyme disease annually. Jama, 310(11), 1110. doi: 10.1001/ jama.2013.278331

- Lambrechts, L., Scott, T. W., \& Gubler, D. J. (2010). Consequences of the expanding global distribution of Aedes albopictus for dengue virus transmission. PLoS Neglected Tropical Diseases, 4(5), e646. doi: 10.1371/journal.pntd.0000646

- Laniak, G. F., Olchin, G., Goodall, J., Voinov, A., Hill, M., Glynn, P.,...Hughes, A. (2013). Integrated environmental modeling: A vision and roadmap for the future. Environmental Modeling \& 1 Software, 39, 3-23.

- Lindgren, E., \& Naucke, T. (2006). Leishmaniasis: Influences of climate and climate change epidemiology, ecology and adaptation measures. In B. Menne, \& K. L. Ebi (Eds.), Climate change and adaptation strategies for human health (pp. 131156). Darmstadt, Germany: Steinkopff Verlag.

- Ludwig, A., Ginsberg, H. S., Hickling, G. J., \& Ogden, N. H. (2015). A dynamic population model to investigate effects of climate and climate-independent factors on the lifecycle of Amblyomma americanum (Acari: Ixodidae). Journal of Medical Entomology, tjv150, 1-17. doi: 10.1093/jme/tjv150

- Mannelli, A., Bertolotti, L., Gern, L., \& Gray, J. (2012). Ecology of Borrelia burgdorferi sensu lato in Europe: transmission dynamics in multi-host systems, influence of molecular processes and effects of climate change. FEMS Microbiology Reviews, 36(4), 837-861.

- Margos, G., Vollmer, S. A., Ogden, N. H., \& Fish, D. (2011). Population genetics, taxonomy, phylogeny and evolution of Borrelia burgdorferi sensu lato. Infection, Genetics and Evolution, 11 (7), 1545-1563.

- Medone, P., Ceccarelli, S., Parham, P. E., Figuera, A., \& Rabinovich, J. E. (2015). The impact of climate change on the geographical distribution of two vectors of Chagas disease: implications for the force of infection. Philosophical transactions of the Royal Society of London. Series B, Biological sciences Royal Society, 370(1665), 20130560. doi: 10.1098/rstb.2013.0560

- Mencke, N. (2011). The importance of canine leishmaniosis in non-endemic areas, with special emphasis on the situation in Germany. Berliner und Münchener tierärztliche Wochenschrift, 124(11-12), 434-442. 
- Miranda, J. E., Navickiene, H. M. D., Nogueira-Couto, R. H., De Bortoli, S. A., Kato, M. J., Bolzani, V. S., \& Furlan, M. (2003). Susceptibility of Apis mellifera (Hymenoptera: Apidae) to pellitorine, an amide isolated from Piper tuberculatum (Piperaceae). Apidologie, 34 (4), 409-415.

- Morán Cadenas, F., Rais, O., Jouda, F., Douet, V., Humair, P. F., Moret, J., \& Gern, L. (2007). Phenology of Ixodes ricinus and infection with Borrelia burgdorferi sensu lato along a northand south-facing altitudinal gradient on Chaumont Mountain, Switzerland. Journal of Medical Entomology, 44 (4), 683-693.

- Murray, N. E. A., Quam, M. B., \& Wilder-Smith, A. (2013). Epidemiology of dengue: past, present and future prospects. Journal of Clinical Epidemiology, 5, 299-309.

- Nonaka, E., Ebel, G. D., \& Wearing, H. J. (2010). Persistence of pathogens with short infectious periods in seasonal tick populations: the relative importance of three transmission routes. PLoS One, 5(7), el 1745. doi: 10.1371/journal.pone.0011745

- Ocampo, C. B. et al. Insecticide resistance status of Aedes aegypti in 10 localities in Colombia. Acta Tropica, 118(1): 37-44, 2011.

- Ogden, N. H., Barker, I. K., Beauchamp, G., Brazeau, S., Charron, D. F., Maarouf, A.,...Lindsay, L. R. (2006). Investigation of ground level and remote-sensed data for habitat classification and prediction of survival of Ixodes scapularis in habitats of southeastern Canada. Journal of Medical Entomology, 43(2), 403-414.

- Ogden, N. H., Radojevic, M., Wu, X., Duvvuri, V. R., Leighton, P. A., \& Wu, J. (2014). Estimated effects of projected climate change on the basic reproductive number of the Lyme disease vector Ixodes scapularis. Environmental Health Perspectives, $122(6), 631-638$.

- Ostfeld, R. S., \& Brunner, J. L. (2015). Climate change and Ixodes tick-borne diseases of humans. Philosophical Transactions of the Royal Society of London B: Biological Sciences, 370(1665), 20140051. doi: 10.1098/rstb.2014.0051

- Oteo, J. A., \& Portillo, A. (2012). Tick-borne rickettsioses in Europe. Ticks and Tick-Borne Diseases, 3(5-6), 271-278.

- Pabón, J. D., \& Nicholls, R. S. (2005). El cambio climático y la salud humana. Biomédica, 25(1), 5-8.

- Parham, P. E., Waldock, J., Christophides, G. K., Hemming, D., Agusto, F., Evans, K. J.,...Michael, E. (2015). Climate, environmental and socio-economic change: Weighing up the balance in vector-borne disease transmission. Philosophical Transactions of the Royal Society of London B: Biological Sciences, 370(1665), 20130551. doi: 10.1098/rstb.2013.0551
- Peniche-Lara, G., Perez-Osorio, C., Dzul-Rosado, K., \& ZavalaCastro, J. (2015). Rickettsiosis: Enfermedad re-emergente en México. Ciencia y Humanismo en la Salud, 2(2), 76-84.

- Pigott, D. M., Bhatt, S., Golding, N., Duda, K. A., Battle, K. E., Brady, O. J.....Hay, S. I. (2014). Global distribution maps of the leishmaniases. elife, 3, e02851. doi: 10.7554/eLife.02851

- Quintero, J., Brochero, H., Manrique-Saide, P., Barrera-Pérez, M., Basso, C., Romero, S.,...Petzold, M. (2014). Ecological, biological and social dimensions of dengue vector breeding in five urban settings of Latin America: A multi-country study. BMC Infectious Diseases, 14(1), 38. doi: 10.1186/1471-2334-14-38

- Radke, E. G., Gregory, C. J., Kintziger, K. W., Sauber-Schatz, E. K., Hunsperger, E. A., Gallagher, G. R.,... Blackmore, C. G. (2012). Dengue outbreak in key west, Florida, USA, 2009. Emerging Infectious Diseases, 18(1), 135-137.

- Ready, P. D. (2008). Leishmaniasis emergence and climate change. Revue Scientifique et Technique (International Office of Epizootics), 27(2), 399-412.

- Rebollar-Téllez, E. A., Tun-Ku, E., Manrique-Saide, P. C., \& Andrade Narvaez, F. J. (2013). Relative abundances of sandfly species (Diptera: Phlebotominae) in two villages in the same area of Campeche, in southern Mexico. Annals of Tropical Medicine and Parasitology, 99(2), 193-201.

- Roberts, M. G., \& Heesterbeek, J. A. P. (2013). Characterizing the next-generation matrix and basic reproduction number in ecological epidemiology. Journal of Mathematical Biology. 66(4-5), 1045-1064.

- Rolandi, C., \& Schilman, P. E. (2012). Linking global warming, metabolic rate of hematophagous vectors, and the transmission of infectious diseases. Frontiers in Physiology, 3, 75. doi: 10.3389/ fphys.2012.00075

- Romer, Y., Nava, S., Govedic, F., Cicuttin, G., Denison, A M., Singleton, J.,...Paddock, C. D. (2014). Rickettsia parkeri rickettsiosis in different ecological regions of Argentina and its association with Amblyomma tigrinum as a potential vector. The American Journal of Tropical Medicine and Hygiene, 91 (6), 1156-1160.

- Schofield, C. J., \& Galvão, C. (2009). Classification, evolution, and species groups within the Triatominae. Acta Tropica, $110(2$ 3), 88-100.

- Shuman, E. K. (2010). Global climate change and infectious diseases. The New England Journal of Medicine, 362, 1061-1063.

- Singh, N., Kumar, M., \& Singh, R. K. (2012). Leishmaniasis: current status of available drugs and new potential drug targets. Asian Pacific Jounal of Tropical Medicine, 5(6), 485-497. 
- Slesak, G., Inthalath, S., Dittrich, S., Paris, D. H., \& Newton, P. N. (2015). Leeches as further potential vectors for rickettsial infections. Proceedings of the National Academy of Sciences, 112 (48), E6593-E6594.

- Stanek, G., Wormser, G. P., Gray, J., \& Strle, F. (2012). Lyme borreliosis. The Lancet, 379(9814), 461-473.

- Süss, J., Klaus, C., Gerstengarbe, F. W., \& Werner, P. C. (2008). What makes ticks tick? Climate change, ticks, and tick-borne diseases. Journal of Travel Medicine, 15(1), 39-45.

- Tánczos, B., Balogh, N., Király, L., Biksi, I., Szeredi, L., Gyurkovsky, M.....Farkas, R. (2012). First record of autochthonous canine leishmaniasis in Hungary. Vector Borne Zoonotic Disease, 12 (7), 588-594.

- Trájer, A. J., Bede-Fazekas, A., Hufnagel, L., Horváth, L., Bobvos, J.,...\& Páldy, A. (2013). The effect of climate change on the potential distribution of the European Phlebotomus species. Applied Ecology and Environmental Research, 11 (2), 189-208.

- Tsetsarkin, K.A. et al. Chikungunya virus: evolution and genetic determinants of emergence. Current Opinion in Virology, 1(4): 310-317, 2014.

- Tun-Lin, W., Burkot, T. R., \& Kay, B. H. (2000). Effects of temperature and larval diet on development rates and survival of the dengue vector Ae. aegypti in north Queensland, Australia. Medical and Veterinary Entomology, 14(1), 31-37.

- Van Bortel, W., Dorleans, F., Rosine, J., Blateau, A., Rousset, D. Matheus, S.,...Zeller, H. (2014). Chikungunya outbreak in the Caribbean region, December 2013 to March 2014, and the significance for Europe. Euro Surveillance: bulletin Europeen sur les maladies transmissibles-European communicable disease bulletin, 19(13). pii: 20759

- Walker, D. H. (2016). Changing dynamics of human-rickettsial interactions. The American Journal of Tropical Medicine and Hygiene, 94(1), 3-4.

- WHO (World Health Organization). (2009). Leishmaniasis: background information. A brief history of the disease [Datos en línea]. Recuperado el 23 de octubre de 2016, de http:// www.who.int/leishmaniasis/en/

- Wright, N. A., Davis, L. E., Aftergut, K. S., Parrish, C. A., \& Cockerell, C. J. (2008). Cutaneous leishmaniasis in Texas: a northern spread of endemic areas. Journal of the American Academy of Dermatology, 58(4), 650-652.

- Yang, H. M., Macoris, M. L., Galvani, K. C., Andrighetti, M. T., \& Wanderley, D. M. (2009). Assessing the effects of temperature on the population of Aedes aegypti, the vector of dengue. Epidemiology \& Infection, 137(08), 1188-1202. 